\title{
TRAVEL ROUTE PLANNING AND TRACKING APPS
}

Artem D. Dymkov,

IRIS Association, Vienna, Austria

DOI: |0.36724/2664-066X-202I-7-2-22-3|

\begin{abstract}
The quality of a tourist trip depends on many factors, and one of the essential is route consistency route. This geographical factor influences the popularity, usefulness, efficiency and tourist travel safety. Drawing up logical routes requires not only a maximum of regional information, but also the developer experience. The creation of clear and convenient routes will allow for more organized tourism activities and reduce the chaotic load on the natural environment. Based on the analysis of applications for planning and tracking routes, this article focuses on the methodology for calculating the time of passage of multi-day cycling routes in main categories and solve the problem of symbiosis between geography and tourism practice. Article will talk about programs that will help you create a route and stick to it along the way.
\end{abstract}

KEYWORDS: 3D mapping, outdoor navigation, cycling routes,

adaptive planning 
There are many useful apps and gadgets for travelers. In this section, author will talk about programs that will help you create a route and stick to it along the way $[3,4]$.

AllTrails provides detailed and hand-picked hiking maps. All maps have reviews, photos from the huge community of the application. The database contains more than 100,000 routes filtered by the required parameters. Use this app for hiking, cycling and running around the world. Download the route of your hike, walk, run or mountain bike ride to your GPS device. Looking for a good place for camping or hiking? The AllTrails community is a great place for inspiration. Download offline maps and start your outdoor adventure.

- $\quad$ AllTrails has the largest collection of GPS trail maps, topographic maps, and downloadable offline maps for trails in national parks and your area.

- Discover the street with new mountain bike, jogging or hiking trails, with reviews and ratings from the hikers, cyclists and trail runners community.

- $\quad$ Find a hike, mountain bike ride, or trail that's perfect for your fitness level and experience.

- Record your outdoor adventures with the AllTrails GPS activity tracker.

- Turn your phone into a GPS activity tracker and follow your trail so you don't get lost while walking outdoors.

- $\quad$ View stats for walking, running and cycling at a glance.

- $\quad$ Share your latest hiking, running and mountain biking with your friends on Facebook, Instagram and WhatsApp.
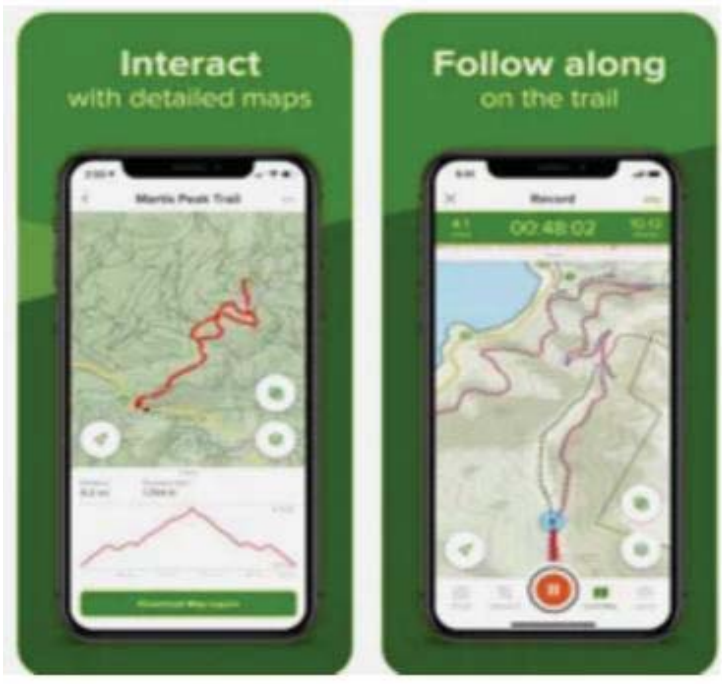

With this application, you can record your movements during hikes in automatic mode, as well as create your own routes and maps. Upon completion, the statistics can be viewed.

Komoot will help you plan your mountain bike route. Offline voice navigation is supported. There is a large database of ready-made routes.

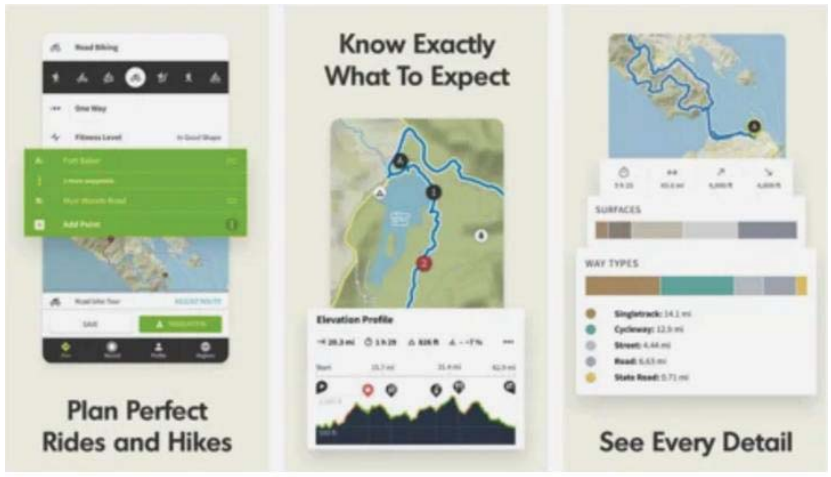

The Komoot app allows you to motivate yourself and makes you run, walk, ride a bike. This application provides all the necessary indicators, and cycling becomes more interesting and brighter. If you want to receive statistics and a lot of other information about your sports activities, then I recommend using this program.

Statistics allows you to get information about what has been done, how much passed, and so on. It is worth stopping here in more detail and talking about what the application gives out as information. Let's start with the distance - the application counts how many meters, or kilometers, you drove. This is important, because just skating thoughtlessly quickly gets boring and you want some kind of records - to drive 20,40 or 50 kilometers. The program counts all this and then shows it at the end of the trip, it is very convenient and helps to take care of yourself. There you can also see the duration of the trip, average speed and calories that you burned during the trip. A very useful thing, you can always look at the results at the end of the trip and draw conclusions - how did you ride in comparison with other days.

The application provides a rather interesting function of choosing a route along which you will go later. This is a very cool function, in fact, you just sit on the sofa and choose where and where you want to go, immediately see the distance and figure out if you can overcome it or not, then get on your bike and leave. The application not only shows on the map where you are driving, but can also act as a navigator.

For cycling routes, the type of road is displayed. Points of interest and elevations have been mapped by the community. Trips can be automatically tracked in the app and shared with friends.

Of course, there were some drawbacks. To begin with, the battery lasts for a very short time and if you want to use its navigator during a trip, it is better to immediately charge the gadget to $100 \%$ and even a portable battery will not be superfluous. There are also problems with navigation on the ground - you are driving, but the application shows that you are standing, and then abruptly throws you to the desired point without adding the distance traveled. This happens very rarely, but it does happen and it is not entirely pleasant.

You can use the application not only for cycling - you can run, ride in the mountains, and so on. There is nothing special here, the functions are, in principle, quite standard, but the ability to plot a route and then follow it with 
navigation is very interesting, because if you travel long distances in an unfamiliar area, it will be very useful.

The Hiking Project offers deep map detail comparable to the printed version. Navigation, hills, photos of places and more. The app suggests places to visit along the route.

The community can supplement the maps with their information or create their own routes.
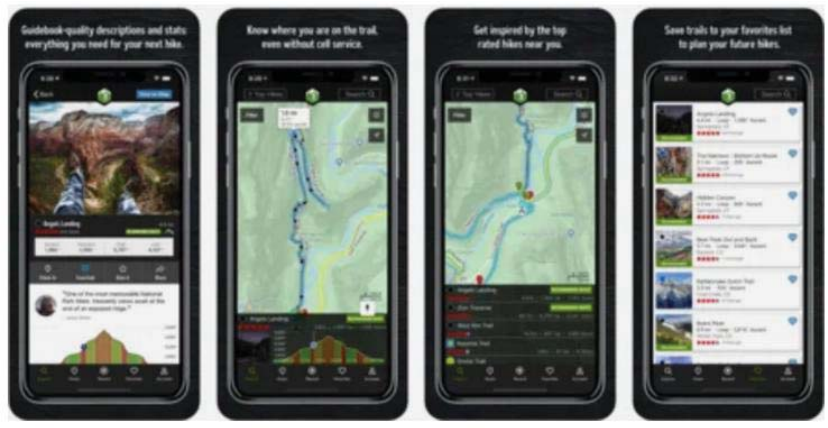

The Hiking Project is revising maps and travel guides. Like a printed route map, the app shows you all known routes. Complete with elevation profiles, full GPS routes, photos and more. As a guide, the program shows you the best recommended hikes that use these routes. Highlights, challenges, special features to look for, local contacts, and even a Virtual Hike so you can experience everything before you hit the road.

If you love exploring outdoors, having a hiking app is equally important as having survival gear to tackle the obstacles that befall your adventure. Besides browsing for interesting trails, a good hiking app helps you navigate safely, alerts your loved ones about your whereabouts, and traces your route accurately.

When it comes to best apps for hiking, you have many options. We have reviewed some famous hiking apps like Komoot, Outdooractive, and AllTrails in our previous articles. Today, we detail the features, pros, and cons of Hiking Project, another leading name in the market.

Hiking Project is famed for its large collection of hiking trails from around the world. Given the similarity of features, it's mostly compared with AllTrails and renowned for its global coverage of hiking trail information.

Hiking Project, as you might have gathered already, is an app solely dedicated to hikers. It was created by REI, a brand renowned for outdoor gear. The app is equipped with details on hiking trails worldwide and provides some handy features to help you figure out the most suitable trail for you.

Users can contribute to the database by providing details on local hiking trails for others to benefit from it. Hiking Project boasts of providing guide-book quality trail information and navigation tips by reviewing all user submissions to ensure they are accurate and reliable.

Hiking Project is available for free of charge. It is available in both website and mobile versions (Android and iOS compatibility). It's free, it doesn't contain advertisements either.
The app opens to a map, and you have to click on the arrow to specify your location and move the map around to find trails located nearby. Hiking Project has over 65,000 trails from around the world in its database, similar to AllTrails.

If you click on a trail, you can read the description of the trail, elevation characters, difficulty level, ratings, additional information, reviews left by previous hikers, and other trails found nearby. There's not a ton of information, but enough to give you the overall picture.

The app offers four main types of apps, namely terrain, satellite, OSM, and NeoTreks. You can download the maps beforehand and use them for navigation even when there's no cell service in the trail. All you have to do is download the GPX file and upload it to a GPS supporting device. You can also trace your route in the airplane mode and check your progress, ensuring you don't go off track.

You can submit information on new trails with a review and pictures. It will be published after being reviewed for authenticity. Additionally, you can also leave comments, ratings, and updated information on existing trails.

Although Hiking Project is not loaded with extra features, it offers a simple, clean, and organized user interface that's easy to navigate. So, if what you are looking for is a basic app with no-frills to support your hiking adventures, this is it.

Free outdoor apps are often blamed for sloppy features, but Hiking Project really stands out in this case. It offers some great and reliable features for an app that's free of charge. Best of all, it doesn't spam your interface with advertisements and diminish your experience.

If you are someone in the U.S, rest assured that you will have a lot of trail options for hiking. There is a lot of options for users from other countries, and it keeps increasing with time!

Cell reception is often unreliable when you are out in the wild, and you need a reliable app to help you complete the hike safely. Hiking Project has in-built GPS to trace your route and keep you updated about your location.

Use of the app can drain your battery faster even when using it on airplane mode, and it's one of the common complaints we found out about the app. If you are using Hiking Project for tracking, we recommend you take a power bank or satellite charger with you.

Compared to other leading outdoor apps like AllTrails, the features offered by Hiking Project are pretty limited. You don't have access to additional features like real-time overlays and off-route notifications - all of which are offered by AllTrails premium.

Hiking Project also doesn't offer the option of searching for trail options using filters, difficulty levels, and activity types.

Many Hiking Project reviews said it's a great app for beginners looking for day hikes. So, it may not be the ideal choice for serious hikers looking for advanced features like route building. That being said, you can use it to browse for trails located close to you and review their background information. Its offline functionality is 
another major benefit to consider. Overall, it's best to review the above pros and cons before making a decision - ut since it's free of charge, you can always try it first hand and decide as well.

If you need a handy app to plan your nature-bound adventures or trips to anywhere on earth, we recommend you try Pilot. It's a social travel planner that lets you plan trips together with friends, family, and even work colleagues. Say goodbye to clutter, as this is the only app you need to keep all your travel details organized to the dot!

Gaia GPS is a mapping service that offers topographic, road and aeronautical maps of the entire world. They can be downloaded to your device for offline use.

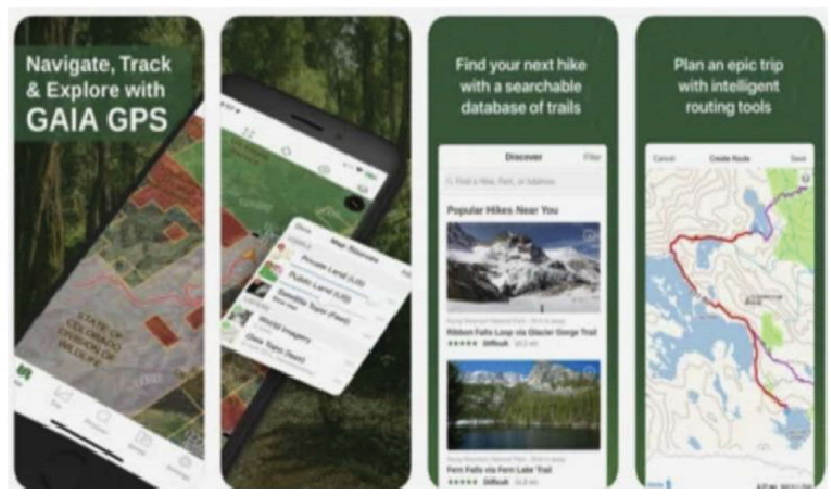

You can plan trips and record GPS tracks for free, view hiking trail maps, hunting parties, and off-road hikes. There is also the option to buy a membership to download maps including topos, aerial imagery, public / private land ownership, and National Geographic. The program also allows you to find your favorite walking route, get directions or find a hunting object. The Gaia GPS app can be used for hiking or even downloading hunting maps, off-road maps and special data such as public and private land ownership. It is possible to sync GPS data and maps seamlessly between all phones, tablets and computers. The program exists not only as a mobile application, but also on the gaiagps.com website. The function of recording trips has been implemented. You can share your travel plans before you hit the road and keep track of them after you return. Gaia GPS works great as a hiking app, hunting app or off-road app.

OpenStreetMap maps are used as a source. There is an online view of topographic maps. For example, to search for the required maps within the United States and Canada, the application offers to use the MyTopo service. Unfortunately, MyTopo cards are only available in the US and Canada. In addition to trips, you can record travel checkpoints and create photos with geo-tags for the convenience of subsequent systematization and search for the desired images and locations. Navigation on the terrain is convenient, the determination of your location is accurate. The search for the desired point or route on the map is fast, you can also view reviews about a particular location. Also, the program implements individual user settings, implying changes in the interface, units of measurement, coordinates and sound effects. The latest version of the application has accelerated the process of loading and showing the display of the necessary routes on the maps, and also eliminated all the bugs that interfered with the normal operation of the application. Overall, objectively speaking, Gaia GPS is one of the most feature-rich navigation apps around.

Once installed, the application provides several functions:

- Route can be created by specifying when to start, when to pause capture, and when to continue.

- In the background, you can view OpenStreetMap or topographic maps with global coverage.

- More than 10 million famous points can be displayed, such as mountains, river crossings, settlements and other points of interest.

- GPS does not depend on internet connection, it still captures, although displaying images only shows what is in the cache.

- $\mathrm{T}$ prevent this, it is possible to save the area as a mosaic of images to display its tileada even offline.

- Stores graphical and statistical counts of each capture point along the route, which can be seen in real time; with data such as geographic coordinates or UTM, current speed, average travel speed, altitude, distance traveled, etc.

The iPad performs much better than the phone because of the size of the display and the ease of using your fingers to interact. The route can then be saved and redeployed for analysis at any time.

At its best, it can run in the background so you can work on other features from iPad or hibernation. At any time, it is activated, and the tour stops or starts a new one without increasing memory or battery consumption.

To display the route on Google Maps, you must register with alltrail.com, including logging in with a Facebook user. Then the route is downloaded from the iPad and the export option is selected. Stored as a new file in My Trips, which can be public or private.

It is possible to view routes using Google Maps layers in the background, be it satellite view, elevation, map or hybrid.

The red line shows the recorded route. On the graph, the traversed profile is displayed in blue, and the speed in kilometers per hour is displayed in orange.

This graph can even be run as a video, although it looks more online.

Decent location accuracy like any browser. It is enough to walk from 3 to 6 meters, although it would be necessary to try to make a static capture, because if you drive a vehicle at a speed of about 50 kilometers per hour, then in some cases you have to check the difference by changing the capture time by distance or seconds.

Of course, the route does not line up so well with the Google Earth photo, not because the device loses accuracy, but because the Google image represents movements between 10 and 20 meters in rural areas away from large cities or with rather irregular topographies 
where the simplicity of the earth model used influenced its georeferencing.

In online mode, the program allows you to add new routes, including clicking on the map and editing by dragging vertices. Another very nice feature is that you can create a new route containing multiple routes. Not bad as it can be sent to GPX to be installed on other devices like Garmin, Magellan, SPOT Satellite Messenger, Blackberry, etc. The program also supports downloading GPX files captured by any GPS navigator. In addition, the route can be exported in $\mathrm{kml}$ so that it can be viewed in 3D in Google Earth.

Wikiloc Outdoor Navigation GPS has over a million routes available worldwide. The routes are categorized by type: hiking, running, cycling, MTB, kayak, skiing and more. You can plan your routes and add photos.

There is the possibility of activating GPS navigation inside the application, tracking the position in real time, the ability to share GPS coordinates.

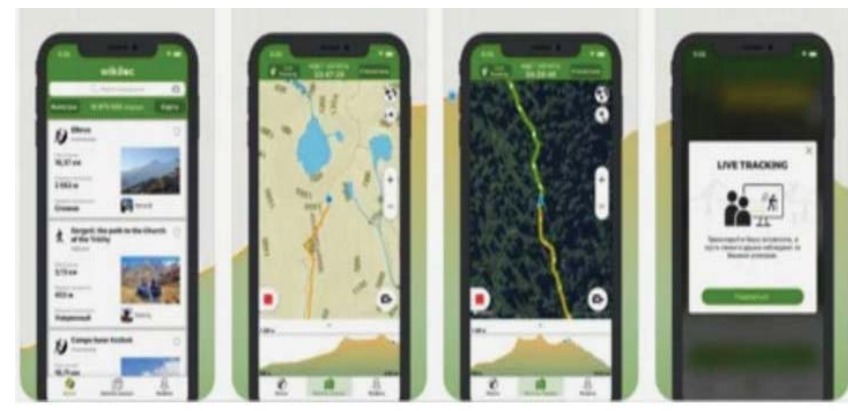

Find the perfect route for your next hike by filtering by distance traveled, altitude gain, or by narrowing down the area of interest using the map.

To find routes and save data in the application, you need the Internet. Record your hikes in nature on a map and get real-time statistics: graphs of speed, distance and altitude; take photos and set directions along the way and upload it all directly to Wikiloc. Offline recording and saving in the app. You need the Internet to send.

Wikiloc Premium allows you to explore and discover the best routes near your location, and follow them with course pointers, compass and audio prompts to alert you if you go astray. No internet needed!
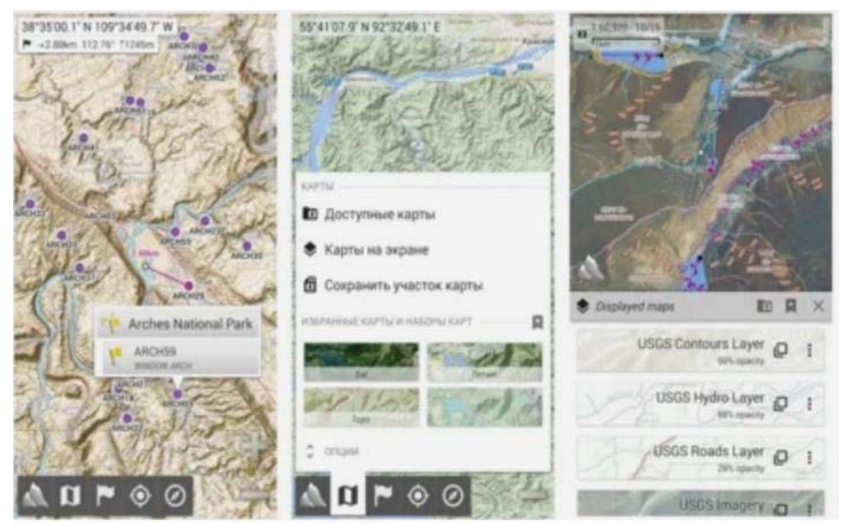

Wikiloc Premium is available as an internal purchase in the free app. Internet is required for searching and saving in the application, as well as for downloading maps for offline use. Once saved, you can track routes offline.

So, you decide who can see your location on the route. Walking the route alone? Activate real-time tracking for your friends or loved ones and they will always know where you are.

Are you racing? Activate real-time tracking so they know where you are or that you are almost at the finish line.

Wikiloc Premium subscription and internet connection required. Subject to the Terms of Use.

Developers have created offline maps so that you can enjoy nature travel around the world, with information about altitude and with features that you need away from the beaten track - on mountain peaks, lakes, streams, springs or in mountain huts.

Once downloaded, the maps are saved on your smartphone and will work even without Internet access. Convenient for areas with poor mobile coverage or when traveling abroad.

Developers strive to provide free offline maps of every corner of the world. If maps of your location are not found, you can inform the developers, and they will try to create maps for your area.

Tell your real friends about your hikes. After finishing the recording of your trip, you can tell about it to your fellow travelers who were with you on the route. Thus, the route will also be displayed in the list of routes they have traveled in their personal profiles.

You can also share your adventures not only with close friends, but also on your social networks by sharing your hikes on Facebook and Twitter.

Download routes from Wikiloc directly to your Garmin, Apple Watch or Suunto. The feature is available on Wikiloc Premium for compatible devices.

AlpineQuest - solution for all types of outdoor activities and sports: hiking, running, hunting, geocaching, off-road and water navigation and much more.
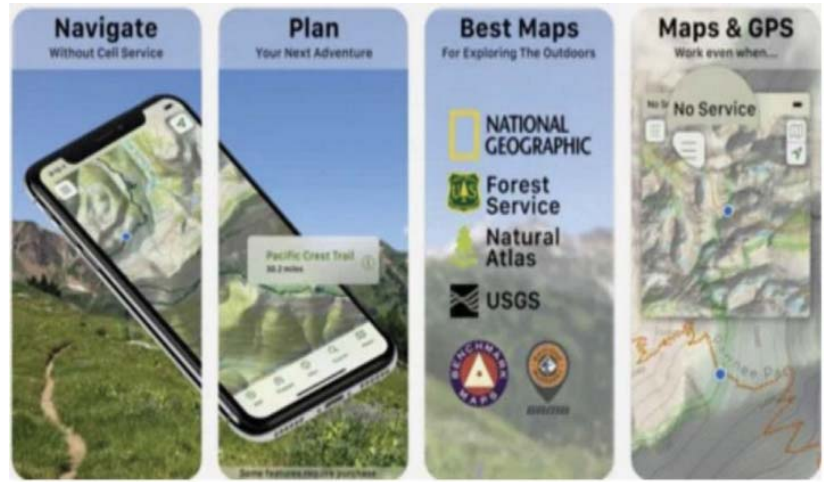

Maps can be saved to the device for offline use. AlpineQuest also supports various map files such as QCT 
and OZFx2. There is support for GPS navigation. You can save and load your waypoints, plan and track routes.

The advantages of this program:

- support for several types of maps (which she knows how to download), between which you can switch in the process;

- fast wor of the interface (smooth scaling, fast rendering);

- support for tracks in GPX format (format of Garmin navigators), KML (format Google Earth);

- recording tracks;

- convenient "estimation" of the distance on the map;

- there is a search for objects (us d by OpenStreetMap, Google, Google Maps).

Minuses:

- no download by region, country, only by the selected area on the map;

- no routing.

Let's consider the main settings of the program:

1) Select the location for saving downloaded maps (phone memory / memory card). It also shows the amount of already downloaded maps and the amount of free space.

2) Disable GPS, if the program is "minimized" (inactive). This checkbox "disable in the background" is already set by default in the program.

3) If GPS is active (trac ng the current position), you can choose to automatically return the screen to the GPS position. That. when scrolling through the map (for example, viewing the road ahead), it itself returns to the place of its current location (and there is no need to "scroll" back). When GPS is off, this setting has no effect - you can flip through the maps as you like.

4) Also the default setting is "Full Screen Mode". Those. when the program is running, the standard Android status bars at the top and buttons at the bottom are not visible. The lack of panels allows you to allocate more space for displaying maps. To make the panels appear in "full screen mode", you need to make a "zoom out" gesture from the edges of the screen (top and bottom)

To select an active map, you need to click "Available maps", then open one of the tabs and select a specific map (its name is in bold). The size of each downloaded map will also be shown (well, the total amount of free space in the memory where the maps are saved).

When downloading, the map type that is currently active will be downloaded. It is necessary to select a rectangular area on the map, with the blue slider at the bottom ("zoom in"), select the desired level of detail, monitor the size of the maps, because at the maximum (13th) detail, their size can be very large, and click the flash drive icon at the bottom right. During the download process, the current download speed, how long to download and the estimated time will be displayed. At the end of the download, an inscription will appear: "completed successfully".
Topo Maps + provides topographic maps from USGS, MapBox, NRCAN/GEOBC and Thunderforest for offline download and use

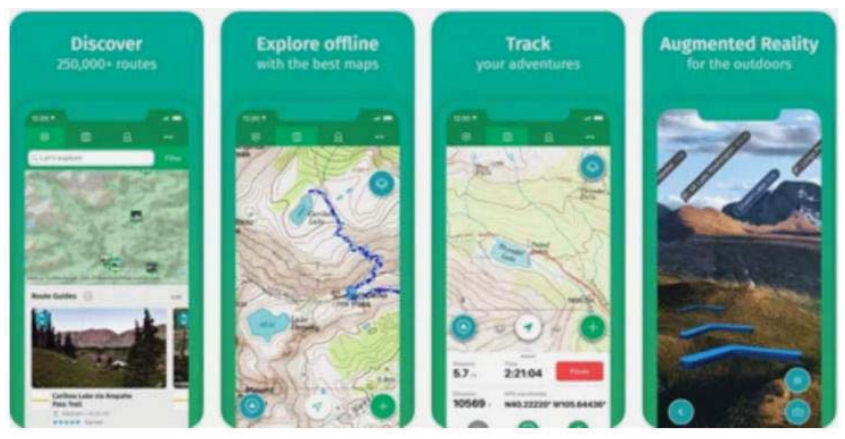

Easily download maps so you can use the maps offline while deep in the backcountry.

Use your iPhone's GPS to view your current location, even when you don't have a cell signal.

GPS and downloaded maps work even without an internet connection so you can use Topo Maps+ deep in the backcountry.

Add waypoints at important locations, like where you parked, so you can navigate to those locations.

Topo Maps+ was designed for serious hikers who love to go deep into backcountry and get as far away from a cell signal as possible. Whether you enjoy pounding out the miles day after day or relaxing at high alpine lakes, Topo Maps+ can help you get there and back again.

Trails Illustrated maps are the most trusted and popular recreation maps available. The USGS 7.5 minute topographic maps are the gold standard for backcountry maps in the USA.

Topo Maps+ has maps for any adventure you want to go on:

- NRCAN Topo Maps cover all of Canada.

- Glacier Topo is a beautiful world wide topographic map with hiking and biking trails.

- $\quad$ Thunderforest is a world wide topographic map with hiking, biking, and skiing trails.

- $\quad$ LINZ Topo Map is the the ultimate backcountry map for New Zealand.

- Satellite Map lets you see satellite imagery for the entire world.

Topo Maps+ has trail data for over 500,000 trails. Just tap on a trail to see distances and elevation profiles. Don't want to exactly follow the trail, no problem. The trails interact with your own traced routes so you can easily explore custom routes. In the wild, trails are not isolated segments between two points, they are a network of interconnected paths with numerous possible routes. Topo Maps+ lets you explore any possible route through a trail network.

Easily trace new routes right on the map and see the routes distance and elevation profile. Create custom routes from trail networks. The trace will snap to the trails. Create waypoints for your trip before you head out. Check out the distance and elevation profile along a trail between any two points. Keep everything for you trip 
organized by simply tagging it. Share all of the routes and waypoints for your trip with your friends. Bring peace of mind, maps \& GPS work offline. Topo Maps+ for iPhone has a deep feature set to help you plan, prepare, and go deeper into the backcountry. Topo Maps+ gives you the tools you need to plan and be prepared for you next backcountry adventure.

Use all of the same features on your iPad and iPhone. Plan your trip on your iPad and then use your data on your iPhone. Topo Maps+ can sync your data between your iPhone and iPad.

View your offline maps and current location just by glancing down at your wrist. You no longer need to pull your iPhone out of your bag to check your position.

ViewRanger is a collection of route guides and topographic maps for travel planning.

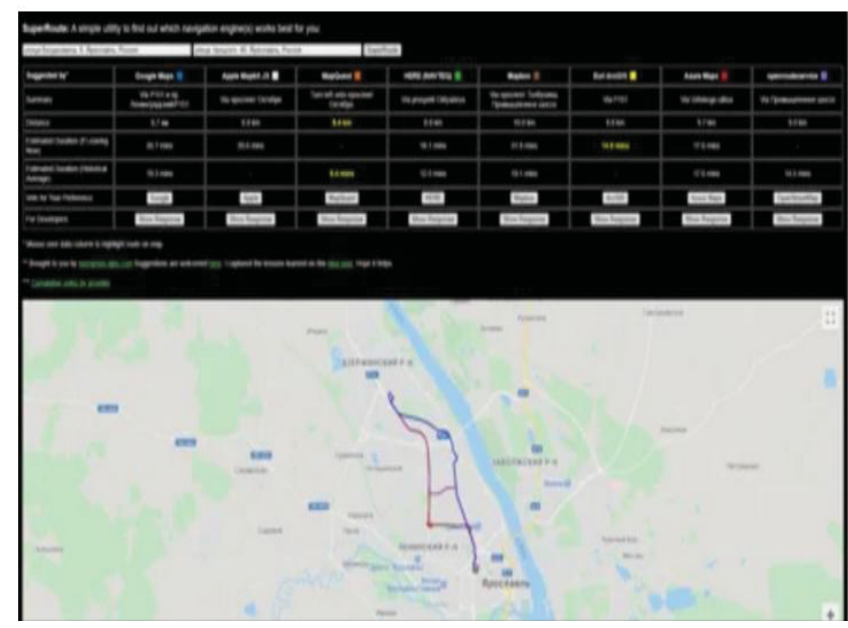

If in cities it is possible to use online maps without any problems, then outside the city, where there may not be a stable reception of mobile networks, the situation is changing. When looking for an app with maps that could show not just something like "you are here in the middle of the forest", but trails and dirt roads can be found on the Android version of ViewRanger. In 90\% (or even more) of cases, all the necessary information is displayed (trails, swampy / wet areas on them, etc.)

Of course, online maps do not offer specialized travel products, but in this case they are mentioned among the most common applications for navigation on mobile devices.

Moving on. Trails are, of course, good. Well, what if there is no stable mobile Internet? With the help of ViewRanger, you can download maps to a device without a $3 \mathrm{G}$ module, but with GPS support, the required area of the map and follow it. Distances and crossroads of trails are reflected quite correctly, it is quite possible to walk on such a "map". But, on the ViewRanger maps, far from all the trails available on the terrain are shown. That is, sometimes you can "cut" rather well, but ViewRanger does not show it.

There are also paid cards. In total, 7-8 free maps are available in the application. There are also additional useful functions. This application can be used according to the scheme "looked at the landmarks on the maps before leaving - opened the application several times so as not to miss a turn - calculated the approximate mileage traveled."

There is a quite stable, low-key application with a good free map + the ability to save fragments of it for offline orientation.

The ViewRanger GPS software, unlike most existing navigators, is a full-fledged tracker and navigation service for building routes, which is focused more on users who love outdoor activities, tourism and travel. Plan your trips quickly and easily, build challenging tourist routes and explore the world with the program. Moreover, the maps are absolutely free, and the main feature is that all streets and sections displayed on the map are displayed in the language of the country where they are located. The ViewRanger GPS interface itself, unfortunately, is presented in English, but it will not be difficult to figure it out.

The advantages of this navigator include fully autonomous operation, even if your gadget has lost the mobile signal. Searching for satellites and displaying your location is almost instantaneous. Any selected area can be saved as a separate offline map and used at any time. It is possible to quickly build and record a route.

Also, while driving, statistics on speed, distance traveled and other useful data are kept and recorded.

You can use the camera to locate peaks, places, and other points using augmented reality. There are threedimensional air tours 3D Flyover. BuddyBeacon helps you share your location with family and friends in real time.

Google Maps app will start directing drivers along routes estimated to generate the lowest carbon emissions based on traffic, slopes, and other factors, the company announced on Tuesday. Google, an Alphabet Inc unit, said the feature would launch later this year in the US and eventually reach other countries as part of its commitment to help combat climate change through its services. Unless users opt-out, the default route will be the "ecofriendly" one if comparable options take about the same time, Google said. When alternatives are significantly faster, Google will offer choices and let users compare estimated emissions.

Google said it derives emissions relative estimates by testing across different types of vehicles and road types, drawing on insights from the U.S. government's National Renewable Energy Lab (NREL). Road grade data comes from its Street View cars as well as aerial and satellite imagery. The potential effect on emissions from the feature is unclear. But in a study of 20 people at California State University, Long Beach, university researchers last year found participants were more inclined to consider carbon emissions in route selection after testing an app that showed estimates.

Google's announcement included additional climatefocused changes. From June, it will start warning drivers about travel through low emissions zones where some vehicles are restricted in Germany, France, the 
Netherlands, Spain, and the UK. In the coming months, Maps app users will be able to compare car, biking, public transit, and other travel options in one place instead of toggling between different sections.

We often use various navigation services. But how can you be sure that Google Maps really does route better than Apple? Or vice versa.

SuperRoute will help you compare the quality of building a route between Google, Apple, MapQuest, Mapbox, HERE, Esri, AzureMaps, OpenRouteService.

To compare routes, you just need to go to service website and enter addresses of origin and destination. Then a map with routes and information about their quality will be displayed. The comparison includes length of journey, duration when departing now, and historical average duration.

Naviki - удобная и практичная программа для велосипедистов
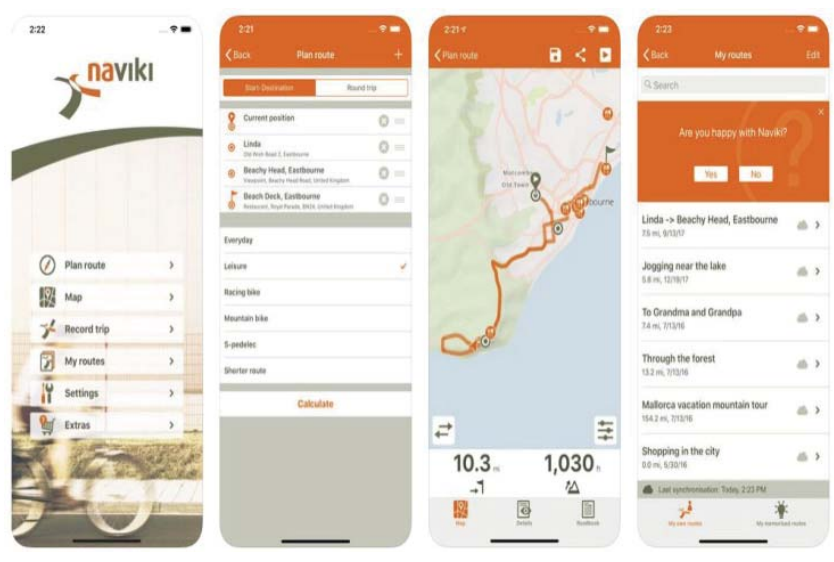

The application will allow you to comfortably plan your cycling trips, raise routes on the map, use navigation.

The Naviki app will record your rides and sync them to www.naviki.org. You will collect all your routes in one place.

Mark the beginning of the route and its end - and get a laid route with the necessary information.

You will see all the necessary information on the display. Audio instructions are not distracting during the ride. If you accidentally (or not accidentally) deviated from the planned route, the program will automatically create a new route.

The program itself will choose the best route, taking into account the road surface, the straightness of the route, the shortest distance and the safety of movement.

Программа The program will lay out individual mountain routes, in which it will take into account the isolation of the track, designated MTB routes, routes in the bosom of picturesque nature, it will choose tracks with the appropriate surface suitable for racing, secondary, unloaded roads - the trips will be as fast, comfortable and safe as possible.

Also, the program will provide you with special safe routes for e-bikes at speeds up to 30 miles per hour and will build various alternative circular routes.
The program has a collection of attractions that will decorate your trip along a particular route.

Download Naviki maps to your device and use them without going online. The program displays the profile of the route height: high, low points and total height.

Maximum speed, average, distance covered, distance to the end of the route are always at your disposal.

The program via Bluetooth will show the distance to the next turn and navigation arrows on the display screen. It is easy to record routes - press the button and the route will be recorded and saved in your personal cloud of the program. All statistics of your trips will be posted on www.naviki.org

The program makes step-by-step instructions on the routes of all randomly chosen ones.

Another very popular application among cyclists is Locus Map Free - Outdoor GPS. Here you can also download maps to your device and then use them without an Internet connection. The first three offline maps will be absolutely free.

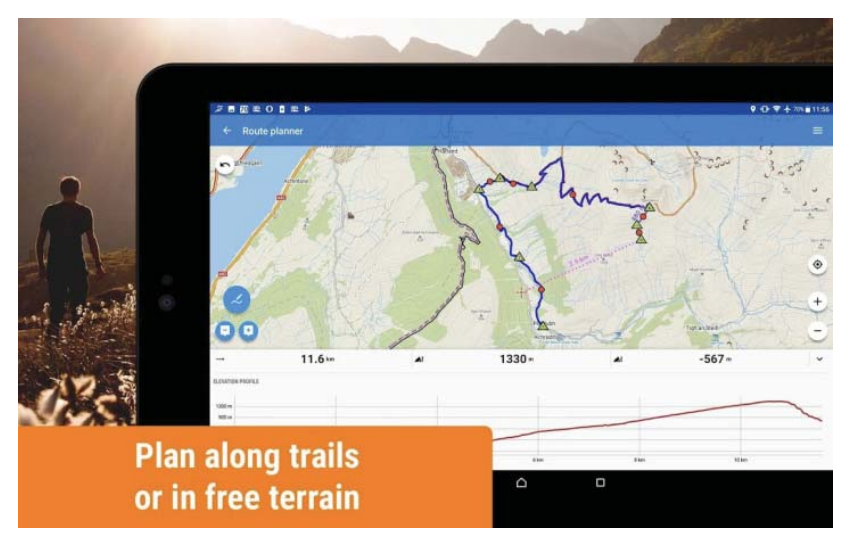

The app is available for free download. But if you want to use additional functions, such as statistics recording or full Bluetooth support, you can buy the Pro version.

Every selection of the best cycling apps will feature this familiar name - Strava.

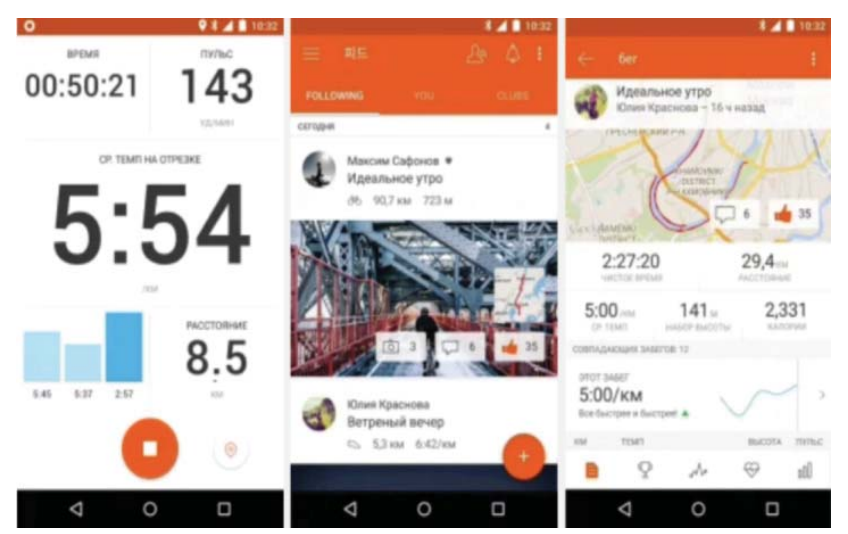

At first glance, this is an unremarkable tracker of routes, speed and other data that athletes love to track. However, as it turns out, Strava is a whole social network 
that allows you to follow the results of your friends and compete with them. In addition, the application has the ability to sync with a variety of wearable equipment. Among them are the budget Amazfit from Xiaomi, sports models from Garmin, Suunto, as well as smart watches based on the Wear OS platform from Google. For iPhone users, there is support for the Apple Watch.

Another important aspect is the routing function in the application. While its support is implemented only through a computer, in the future the developers promise to implement it on mobile devices, however, it is much more convenient to plan a trip route from a computer. During the trip, there are voice prompts and voice navigation. So, we can say that Strava is a whole platform with many different features that will be useful not only for cyclists, but also for runners. The app is free and available on Google Play. No advertisements, however, there are optional transactions for additional features. And without them, you can fully use the application.

Any cyclist at least once thought about how interesting it would be to ride a route created by another user. And this idea is brought to life by the BikeMap application. Here you will find a built-in map, the ability to use the program without the Internet, a convenient speedometer and a personal account. To gain access to all functions, you need to go through a simple registration. It's even good, because when you change your phone, you can restore your progress and statistics.
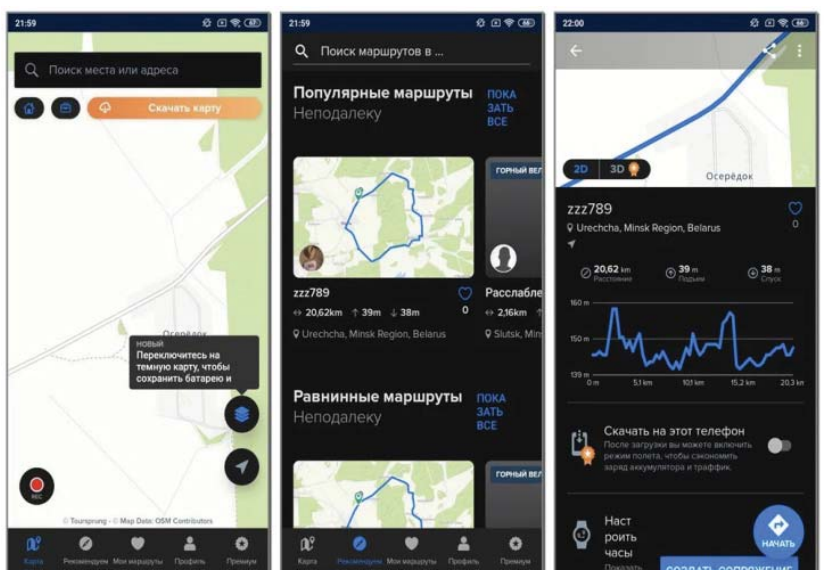

The start screen contains a map of your area. If it is not displayed, then check if GPS is activated. And the most interesting tab is "Recommended". There are other people's routes for your area, and they are even divided into categories. Do you like to ride on a bad road? Take a look at the Plain Routes menu. Do you prefer evening bike rides around the city? Go to the "Popular Routes" section.

The map will show the total duration of the route, the ratings of other users and the name of the author. Just save the card to your phone and use it even without an internet connection. And if you have a smartwatch, then you can easily link it to the application.
For anyone, even a novice cyclist, the most important thing is the accuracy of measurements and, of course, the availability of a convenient map with a route. All of these features can be found in the Strava app, but not every user likes it. A relatively new program was discovered on Google Play, which received a simple name Ride with GPS.

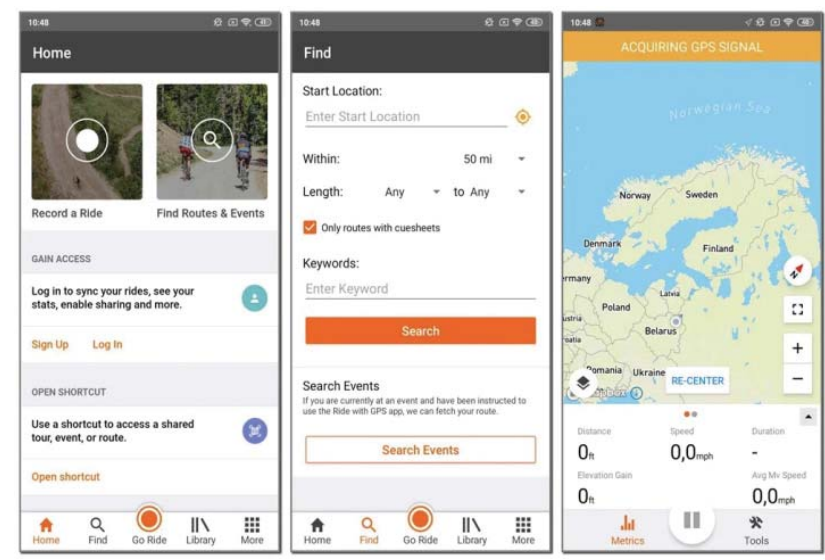

It is a handy utility with a built-in map, ready-made routes and data synchronization. But the main drawback for the Russian-speaking population is a completely English-language interface. However, after a few days of using the program, you will not even read the names of the tabs, because everything will become intuitive.

To work with the application, you need to perform a simple registration by entering your email address and password. And after that you can start the virtual computer and start your trip. A map will be displayed on the screen, and just below a block with all the data. Information about the current speed, distance traveled and travel time is indicated here. Optionally, you can collapse the map and turn the program into a full-fledged bike computer.

Each of us at least once in our life found ourselves at a crossroads and did not know how to return to civilization. Next time, before driving on an unknown route, do not forget to download TrailForks before leaving your home: it will save you a couple of hours of wandering through the woods or along the slopes of the mountains.

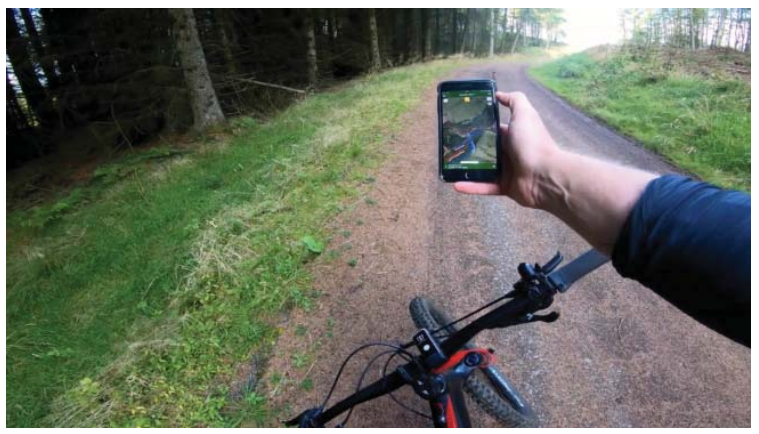

Drawing on information from other cyclists, the program boasts detailed maps of more than 161,000 routes around the world, real-time geolocation + 
information on attractions. In case something goes wrong, there is an alarm function that transmits your exact GPS coordinates and the name of the nearest settlement. It is extremely important if you like to drive alone.

BikeComputer - another version of the cycling computer, which, unlike its analogues, does not require any registration. It also allows you to track physical activity and get detailed statistics for each race. The ability to get directions is available both online and for maps downloaded through the application.

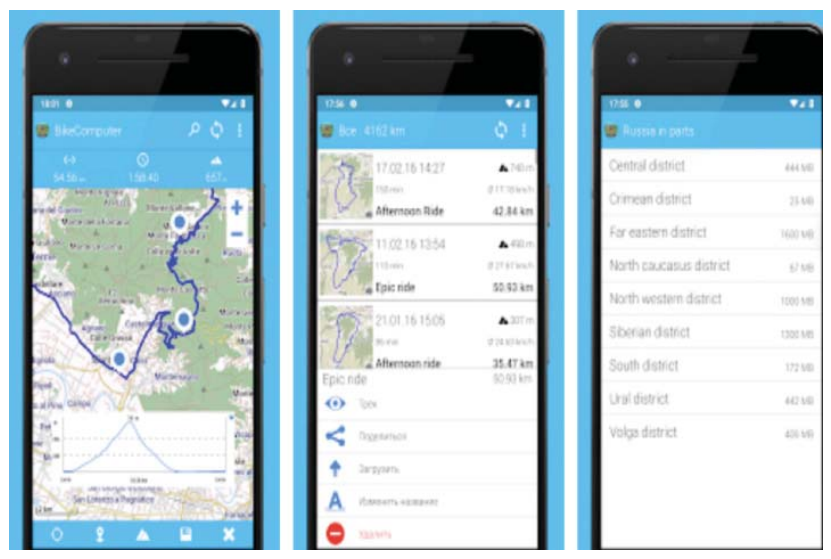

Viewranger is useful for those who prefer to ride a bike away from noisy cities and busy highways. Recommended for all bike travelers and cyclists who value adventure and nature. The Viewranger app relies on the free OpenCycle map that covers the entire world, so you can get directions for cycling where other apps fail. Viewranger offers 20 detailed country maps for an additional fee. The application stores maps on the phone, and uses the built-in GPS for navigation, so there is no need for a constant Internet connection - excellent functionality for cyclists who prefer wild forest or mountain trails.

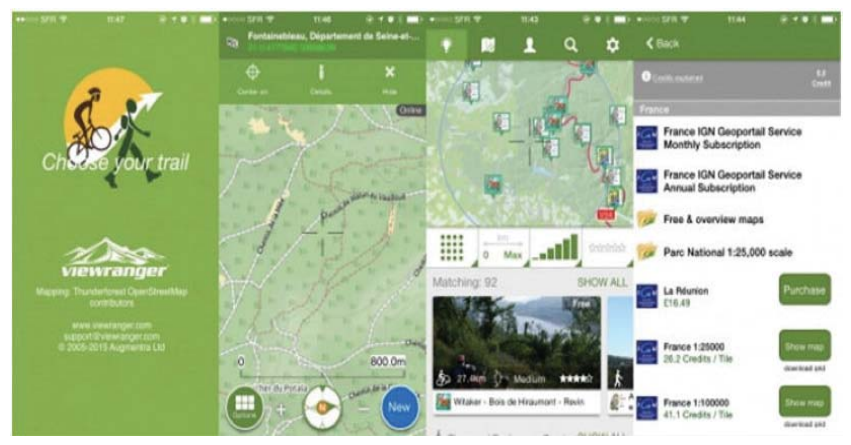

The app allows you to create routes and share them with other cyclists, as well as download tracks from other cycling enthusiasts. The Viewranger has a real-time location tracking function called Buddy Beacon, which allows you to show your current coordinates on the map for friends or generally for all users of the application. The Buddy Beacon bike app is available for free on both iOS and Android. There are in-app purchases.

\section{CONCLUSION}

In this article analysis applications for planning and tracking routes, which allows to develop clear and wellconstructed tourist routes and solve specific problems, including taking into account weather conditions and terrain. Modern sports tourism is being transformed under the influence of global tourism trends. Meanwhile, creation of clear and convenient routes will allow for more organized tourism activities, improve control over "wild" tourism and reduce the chaotic pressure on natural environment. It is important to understand, evaluate and preserve those of its strengths that allowed this area to develop successfully and solve problem of symbiosis between geography and tourism practice.

\section{REFERENCES}

[1] S. S. Dymkova, "Coniunction and synchronization methods of earth satellite images with local cartographic data," 2020 Systems of Signals Generating and Processing in the Field of on Board Communications, Moscow, Russia, 2020, pp. 1-7, doi: 10.1109/IEEECONF48371.2020.9078561.

[2] S. V. Khadonova, A. V. Ufimtsev and S. S. Dymkova, "Wide application innovative monitoring system with personal smart devices," 2020 Systems of Signal Synchronization, Generating and Processing in Telecommunications (SYNCHROINFO), Svetlogorsk, Russia, 2020, p. 1-5.

10.1109/SYNCHROINFO49631.2020.9166115.

[3] S. S. Dymkova and A. D. Dymkov, "Synchronizing of moving object with novel 3D maps imaging," 2020 Systems of Signal Synchronization, Generating and Processing in Telecommunications (SYNCHROINFO), Svetlogorsk, Russia, 2020, pp. 1-5, doi 10.1109/SYNCHROINFO49631.2020.9166029.

[4] S. S. Dymkova and A. D. Dymkov, "Multifactorial methodology of cycling routes time calculation based on 3D maps," 2021 Systems of Signals Generating and Processing in the Field of on Board Communications, Moscow, Russia, 2021, pp. 1-8. 